\title{
Study of neurinomas with ultrasound contrast media: review of a case series to identify characteristic imaging patterns
}

\section{Lo studio dei neurinomi con mezzo di contrasto ecografico: valutazione dei patterns caratteristici di riconoscimento diagnostico}

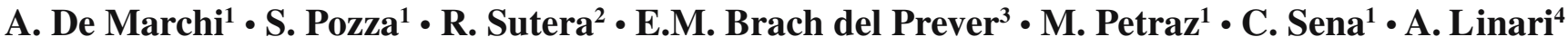 \\ C. Faletti ${ }^{1}$
}

\author{
${ }^{1}$ Dipartimento di Diagnostica per Immagini, UOA di Radiologia Diagnostica, Azienda Ospedaliera CTO-CRF-Maria Adelaide, Torino, \\ Italy \\ ${ }^{2}$ Sezione di Scienze Radiologiche, Di.Bi.Me.F, Università di Palermo, Italy \\ ${ }^{3}$ Dipartimento di Traumatologia, Ortopedia e Medicina del Lavoro, Azienda Ospedaliera CTO-CRF-Maria Adelaide, Torino, Italy \\ ${ }^{4}$ Servizio di Anatomia Patologica, A.O. O.I.R.M. S. Anna, Torino, Italy \\ Correspondence to: S. Pozza, Tel.: +39-011-6933357, Fax: +39-011-6966948, e-mail: simona.pozza@cto.to.it
}

Received: 27 September 2010 / Accepted: 4 October 2010 / Published online: 19 March 2011

(C) Springer-Verlag 2011

\begin{abstract}
Purpose. The aim of this study was to evaluate whether there exists a characteristic distribution pattern of vessels within neurinomas that may be used to characterise this type of lesion by employing a contrast-specific ultrasound technique.

Materials and methods. Between January 2003 and May 2010, 66 suspected neurinomas were evaluated according to their sonographic features (solid fusiform mass with well-defined margins located in direct continuity with the nerve that was not always discernible and heterogeneous as a result of the presence of small cystic areas or calcifications). The lesions were examined using a sonographic contrast medium consisting of sulphur hexafluoride microbubbles and equipment with dedicated contrast-specific software [contrast tuned imaging (CnTI)]. Of these lesions, five were excluded from the analysis because the definitive diagnosis was not available (in two cases, the follow-up was still in progress, whereas in the remaining three, there was no follow-up). Our study, therefore, is based on 61 surgically excised lesions that were confirmed to be neurinomas by histology, which is regarded as the gold standard.

Results. In 41/61 cases (67.2\%), we identified an enhancement pattern that we termed reticular owing to the interweaving of blood vessels, of which two subtypes were identified depending on whether the interwoven vessels were densely or sparsely packed: loose-knit
\end{abstract}

\begin{abstract}
Riassunto
Obiettivo. Scopo dello studio è valutare, mediante l'impiego di una tecnica ecografica contrasto-specifica, se esiste una distribuzione caratteristica dei vasi all'interno dei neurinomi che possa essere usata per una caratterizzazione del tipo di lesione.

Materiali e metodi. Nel periodo compreso tra gennaio 2003 e maggio 2010 sono state valutate nel nostro dipartimento 66 lesioni sospette per neurinoma in base alle loro caratteristiche ecografiche (formazioni solide, fusiformi, a margini netti, in diretta continuità con la fibra nervosa, non sempre riconoscibile, disomogenee per l'eventuale presenza di piccole aree similcistiche o calcificazioni), ed esaminate con mezzo di contrasto (MdC) ecografico, costituito da microbolle a base di esafluoruro di zolfo e apparecchiatura dotata di software dedicato Contrast Tuned Imaging (CnTI) contrasto specifico. Di queste lesioni, 5 sono state escluse dall'analisi in quanto non era disponibile una diagnosi definitiva ( $n=2$ follow-up in corso; $n=3$ assenza di follow-up). Pertanto il nostro studio si basa unicamente sulle 61 lesioni asportate chirurgicamente e confermate come neurinomi all'esame istologico, considerato come gold standard.

Risultati. In 41/61 (67,2\%) casi abbiamo identificato una distribuzione del mezzo di contrasto definita "a reticolo" in base all'intreccio formato dalle strutture vascolari, in cui si riconoscono due sottotipi: "reticolo a maglie larghe" in 18/41 e "reticolo a maglie strette" in 23/41,
\end{abstract}


reticular in 18/41, and tight-knit reticular in 23/41. In 20/61 (32.8\%) cases, we observed a vascular pattern of diffuse heterogeneous enhancement, which was divided into two subtypes based on the presence of one (7/20) or more $(13 / 20)$ avascular areas.

Conclusions. Results showed that all neurinomas studied could be divided into two groups according to the type of enhancement pattern observed: reticular or diffuse heterogeneous.

Keywords Schwannoma - Ultrasound · Power Doppler · Imaging contrast media distinti in base all'aspetto dell'incrocio dei vasi, se più rado o più fitto. In 20/61 (32,8\%) casi abbiamo osservato un diverso pattern di vascolarizzazione, denominato "impregnazione diffusa disomogenea", a sua volta suddiviso in due sottotipi in base alla presenza di una (7/20) o più aree avascolari (13/20).

Conclusioni. I risultati ottenuti in questo studio hanno dimostrato che tutti i neurinomi analizzati possono essere distinti in due gruppi, in base al tipo di pattern vascolare riscontrato: di tipo reticolare o ad impregnazione diffusa disomogenea.

Parole chiave Schwannoma Ecografia Power doppler . Mezzi di contrasto ecografici

\section{Introduction}

Neurinomas, or schwannomas, are benign tumours that originate from the sheath of peripheral nerves, in relation to which they may align themselves either eccentrically or peripherally [1]. When stimulated mechanically, they often give rise to pain that feels like an electric shock [2], a feature that allows for a well-founded diagnostic hypothesis. Sometimes, however, the clinical presentation is doubtful or of scarce significance. These equivocal findings therefore justify the need for ultrasonography (US).

The morphology and echostructure of neurinomas may vary depending on the extent of growth. Recent neurinomas are usually fusiform, hypoechoic and homogeneous [3-5], whereas older ones may exhibit cystic areas and, more rarely, calcifications [1, 6-8]. Usually, a hyperechoic, at times incomplete, peripheral rim is visible, which is made up of the perineurium [5, 9]. High-resolution US (HRUS) performed with high-frequency linear-array probes is an excellent modality for visualising and in some cases differentiating peripheral nerve tumours, as in most cases it allows for the easy recognition of direct continuity between the nerve and the two poles of the lesion on longitudinal sections [10]. Power-Doppler (PD) is an essential complement to baseline US, as the presence of blood vessels within the lesion will influence any diagnostic suspicion [3]. In fact, lesions with similar appearance at baseline US may prove different in nature on colour Doppler US as a result of their vascular characteristics. However, although power Doppler imaging is particularly sensitive to low flows, it is insufficient to provide a real estimate of lesion vascularity.

Recent years have seen the increasing use of contrastspecific US techniques, which optimise recording of the harmonic signal produced by the contrast microbubbles that amplify the signal from the blood vessels [11, 12].

\section{Introduzione}

I neurinomi, o schwannomi, sono tumori benigni che prendono origine dalla guaina dei nervi periferici, rispetto ai quali possono disporsi eccentricamente o perifericamente [1]. Sono spesso accompagnati da sintomatologia dolorosa, tipo "scossa elettrica" [2], qualora sollecitati meccanicamente, caratteristica, questa, che ne permette un sospetto diagnostico fondato; a volte il quadro clinico è dubbio o poco significativo. Tali riscontri non univoci giustificano, quindi, la necessità dell'esecuzione dell'esame ecografico.

La morfologia e l'ecostruttura dei neurinomi può variare a seconda del grado di crescita; quelli di data recente solitamente si presentano fusiformi, ipoecogeni e omogenei [3-5]; quelli di più vecchia data, invece, possono presentare al loro interno areole similcistiche e, raramente, calcificazioni [1, 6-8]. Solitamente si visualizza un orletto periferico, talora incompleto, iperecogeno, costituito dal perinevrio [5, 9]. L'ecografia ad alta risoluzione, ovvero effettuata con sonde lineari ad alta frequenza, costituisce un'ottima metodica per visualizzare e, talora, differenziare i tumori del sistema nervoso periferico, poiché permette agevolmente di dimostrare, nella maggior parte dei casi, la diretta continuazione con la terminazione nervosa ai due poli della lesione, evidenziabile nelle scansioni longitudinali [10]. Il power doppler $(P D)$ costituisce un indispensabile completamento all'esame basale, poiché la presenza di vasi all'interno della lesione condiziona il sospetto diagnostico [3]. Infatti lesioni ecograficamente simili all'esame basale, possono risultare differenti per natura allo studio con modulo colore, grazie alle caratteristiche vascolari. Tuttavia lo studio con PD ̀̀ particolarmente sensibile ai flussi lenti, ma insufficiente a fornire una reale stima della vascolarizzazione.

Da qualche anno è sempre più frequente l'impiego di tecniche ecografiche contrasto specifiche, che ottimizzano la registrazione del segnale armonico prodotto dalle microbolle del mezzo di contrasto (MdC) ecografico, le quali consentono un potenziamento del segnale proveniente dai vasi [11, 
Unlike diffusive contrast media (such as those used in magnetic resonance imaging and computed tomography) that enter the extracellular spaces, US contrast agents remain confined within the vessels, allowing for clear visualisation of even the smallest vascular structures (the parenchymal microvasculature). US contrast agents have a wide range of applications, especially in studying liver and breast focal lesions and in the field of cardiovascular imaging and haemodynamics [13-15]. Few studies have investigated the use of contrast agents in the musculoskeletal system, and no scientific contributions to date have studied the use of US contrast agents for studying neurinomas [16]. The aim of our study was to assess whether there is a characteristic distribution of blood vessels within neurinomas and to attempt to identify a system for their characterisation.

\section{Materials and methods}

Between January 2003 and May 2010, 66 lesions were identified as possible neurinomas on the basis of for their US features (solid, fusiform masses with well-defined margins, at times in direct continuity with the nerve fibre or heterogeneous as a result of the presence of small cystic areas or calcifications). Five of these lesions were excluded from the analysis because a definitive diagnosis was not available ( $n=2$ with follow-up still in progress; $n=3$ without follow-up). Sixty-one lesions were excised surgically and confirmed as neurinomas by histology, which was regarded as the gold standard. All of these were single lesions except in the case of a woman who had two separate lesions, one in her right thigh; the other in her left leg. These 61 lesions therefore form the basis of our study.

The patients ( 35 females, 25 males) were aged between 15 and 90 (mean 47) years. Thirty-eight neurinomas were intramuscular and 23 subcutaneous. Twenty-four affected an upper limb and 35 a lower limb; one was located in the dorsal region and one in the cervical region. Lesion size ranged from $0.9 \mathrm{~cm}$ to $7 \mathrm{~cm}$ (Table 1).

US examinations were performed using an Esaote Technos MPX unit equipped with LA424 14-8 MHz, LA523 13-8 MHz and LA532E 8-3 MHz linear-array probes and dedicated low-mechanical-index (45 kPa) CnTI software, which allows real-time analysis of the signal generated by the contrast medium. All examinations were performed by two operators, both experts in US imaging. Patients were examined in the supine or prone position, depending on lesion location. After an initial baseline US examination, a colour power Doppler evaluation was obtained. In all cases $2.4 \mathrm{ml}$ of US contrast agent consisting of sulphur hexafluoride microbubbles (SonoVue, Bracco, Italy) was administered as a bolus in a peripheral vein, followed by
12]. A differenza dei mezzi di contrasto diffusivi (utilizzati in risonanza magnetica [RM] e tomografia computerizzata [TC]), che si dispongono anche negli spazi extracellulari, il mezzo di contrasto ecografico rimane confinato, per le sue caratteristiche fisiche, all'interno dei vasi, consentendo così una precisa visualizzazione anche delle strutture vascolari più piccole (microvascolarizzazione parenchimale). Il MdC ecografico presenta un ampio campo di applicazione soprattutto in ambito epatico e mammario, per lo studio delle lesioni focali, nel distretto cardio-vascolare e nell'emodinamica [13-15]. In letteratura l'impiego del MdC in ambito muscolo-scheletrico è scarsamente rappresentato e non sono stati attualmente riscontrati contributi scientifici in merito allo studio dei neurinomi con MdC ecografico [16]. Scopo del nostro studio è valutare se esiste una distribuzione caratteristica dei vasi all'interno dei neurinomi e tentare in tal modo una caratterizzazione degli stessi.

\section{Materiali e metodi}

Dal gennaio 2003 al maggio 2010 sono state valutate presso il nostro reparto 66 lesioni che, in base alle loro caratteristiche ecografiche (formazioni solide, fusiformi, a margini netti, talora in diretta continuità con la fibra nervosa, disomogenee per l'eventuale presenza di piccole aree similcistiche o calcificazioni), sono state identificate come probabili neurinomi. Di queste lesioni, 5 sono state escluse dall'analisi in quanto non era disponibile una diagnosi definitiva ( $n=2$ follow- up in corso; $n=3$ assenza di follow-up). Le lesioni asportate chirurgicamente e confermate come neurinomi all'esame istologico, considerato come gold standard, sono state 61, tutte uniche tranne nel caso di una donna che presentava due lesioni distinte, una alla coscia destra e una alla gamba sinistra; è dunque unicamente su queste lesioni che si basa il nostro studio.

I pazienti (35 femmine, 25 maschi) avevano un'età compresa tra 15 e 90 anni (età media 47 anni); 38 neurinomi erano disposti in sede inframuscolare e 23 sottocutanea; 24 interessavano l'arto superiore, 35 l'arto inferiore, 1 il dorso e 1 la regione cervicale; le dimensioni variavano da 0,9 a 7 cm (Tabella 1).

Gli esami sono stati condotti con ecografo Technos MPX Esaote (Biosound Esaote, Inc, Indianapolis, USA), dotato di sonde lineari LA424 14-8 MHz, LA523 13-8 MHz e LA532E 8-3 MHz e di software dedicato (CnTI) a basso indice meccanico $(45 \mathrm{kPa})$, che consente l'analisi in real time del segnale proveniente dal mezzo di contrasto. L'esame è sempre stato eseguito da due operatori, esperti della metodica. Tutti $i$ pazienti sono stati esaminati in posizione supina o prona, a seconda della sede della lesione, inizialmente con esame ecografico basale, successivamente con valutazione colorpower doppler. In tutti i casi è stata somministrata a bolo, in una vena periferica, la quantità di 2,4 ml di MdC ecografico costituito da microbolle a base di esafluoruro di zolfo (SonoVue, Bracco, Milano, Italia), seguita da un lavaggio 
Table 1 Characteristics of patients and lesions

\begin{tabular}{|c|c|c|c|c|c|c|}
\hline Sex & Age & Anatomical site & Location & Pattern & Subgroup & Diameter $(\mathrm{cm})$ \\
\hline $\mathrm{F}$ & 32 & Lower limb & Intramuscular & 2 & A & 1.5 \\
\hline $\mathrm{F}$ & 55 & Lower limb & Intramuscular & 2 & $\mathrm{~B}$ & 4 \\
\hline M & 61 & Lower limb & Intramuscular & 1 & $\mathrm{~B}$ & 4.5 \\
\hline $\mathrm{F}$ & 37 & Lower limb & Intramuscular & 1 & $\mathrm{~B}$ & 1.5 \\
\hline $\mathrm{F}$ & 35 & Lower limb & Intramuscular & 2 & $\mathrm{~B}$ & 6 \\
\hline $\mathrm{F}$ & 45 & Lower limb & Intramuscular & 1 & A & 1 \\
\hline $\mathrm{F}$ & 45 & Lower limb & Intramuscular & 1 & A & 3 \\
\hline M & 90 & Lower limb & Subcutaneous & 2 & B & 2.5 \\
\hline $\mathrm{F}$ & 58 & Lower limb & Intramuscular & 1 & $\mathrm{~B}$ & 3 \\
\hline $\mathrm{F}$ & 58 & Lower limb & Intramuscular & 1 & $\mathrm{~B}$ & 4 \\
\hline M & 68 & Lower limb & Intramuscular & 1 & $\mathrm{~B}$ & 3 \\
\hline M & 50 & Lower limb & Intramuscular & 1 & A & 2.5 \\
\hline M & 64 & Lower limb & Intramuscular & 1 & $\mathrm{~B}$ & 3.5 \\
\hline $\mathrm{F}$ & 23 & Lower limb & Intramuscular & 1 & A & 2.4 \\
\hline M & 37 & Lower limb & Subcutaneous & 1 & A & 2.3 \\
\hline $\mathrm{F}$ & 55 & Lower limb & Intramuscular & 2 & A & 2.5 \\
\hline M & 72 & Lower limb & Intramuscular & 2 & B & 4.3 \\
\hline $\mathrm{F}$ & 30 & Lower limb & Subcutaneous & 1 & $\mathrm{~B}$ & 4 \\
\hline $\mathrm{F}$ & 66 & Lower limb & Subcutaneous & 1 & A & 3 \\
\hline M & 40 & Lower limb & Intramuscular & 1 & $\mathrm{~B}$ & 1.2 \\
\hline M & 22 & Lower limb & Subcutaneous & 2 & B & 3.4 \\
\hline M & 56 & Lower limb & Intramuscular & 1 & B & 2.3 \\
\hline $\mathrm{F}$ & 27 & Lower limb & Subcutaneous & 2 & A & 2.4 \\
\hline M & 80 & Lower limb & Subcutaneous & 1 & B & 1.1 \\
\hline $\mathrm{F}$ & 19 & Lower limb & Subcutaneous & 2 & B & 3 \\
\hline $\mathrm{F}$ & 22 & Lower limb & Intramuscular & 2 & $\mathrm{~B}$ & 4.1 \\
\hline M & 43 & Lower limb & Intramuscular & 2 & B & 2 \\
\hline M & 46 & Lower limb & Subcutaneous & 1 & $\mathrm{~B}$ & 3.5 \\
\hline M & 69 & Lower limb & Subcutaneous & 1 & $\mathrm{~B}$ & 2.5 \\
\hline $\mathrm{F}$ & 29 & Lower limb & Intramuscular & 1 & A & 2 \\
\hline $\mathrm{F}$ & 52 & Lower limb & Intramuscular & 1 & A & 7 \\
\hline $\mathrm{F}$ & 64 & Lower limb & Intramuscular & 1 & B & 0.9 \\
\hline M & 40 & Lower limb & Subcutaneous & 1 & B & 3 \\
\hline $\mathrm{F}$ & 37 & Lower limb & Subcutaneous & 1 & A & 3.6 \\
\hline M & 67 & Lower limb & Subcutaneous & 1 & B & 4 \\
\hline $\mathrm{F}$ & 39 & Upper limb & Intramuscular & 1 & B & 4 \\
\hline M & 48 & Upper limb & Intramuscular & 1 & $\mathrm{~B}$ & 7 \\
\hline $\mathrm{F}$ & 15 & Upper limb & Intramuscular & 2 & A & 2 \\
\hline $\mathrm{F}$ & 26 & Upper limb & Intramuscular & 1 & A & 1 \\
\hline M & 34 & Upper limb & Intramuscular & 2 & A & 2.5 \\
\hline M & 32 & Upper limb & Intramuscular & 1 & A & 2 \\
\hline $\mathrm{F}$ & 43 & Upper limb & Intramuscular & 1 & A & 4 \\
\hline M & 48 & Upper limb & Intramuscular & 1 & A & 2 \\
\hline $\mathrm{F}$ & 73 & Upper limb & Intramuscular & 1 & A & 5 \\
\hline $\mathrm{F}$ & 51 & Upper limb & Subcutaneous & 1 & $\mathrm{~B}$ & 1 \\
\hline M & 54 & Upper limb & Intramuscular & 1 & A & 7 \\
\hline $\mathrm{F}$ & 26 & Upper limb & Intramuscular & 1 & $\mathrm{~B}$ & 3 \\
\hline $\mathrm{F}$ & 58 & Upper limb & Subcutaneous & 1 & A & 2.5 \\
\hline $\mathrm{F}$ & 46 & Upper limb & Subcutaneous & 2 & B & 2.1 \\
\hline $\mathrm{F}$ & 48 & Upper limb & Subcutaneous & 1 & B & 1.5 \\
\hline M & 65 & Upper limb & Intramuscular & 2 & B & 4 \\
\hline $\mathrm{F}$ & 47 & Upper limb & Intramuscular & 1 & B & 2.9 \\
\hline M & 63 & Upper limb & Subcutaneous & 1 & A & 2.7 \\
\hline $\mathrm{F}$ & 43 & Upper limb & Subcutaneous & 2 & $\mathrm{~B}$ & 1.4 \\
\hline M & 51 & Upper limb & Intramuscular & 2 & $\mathrm{~B}$ & 0.9 \\
\hline $\mathrm{F}$ & 32 & Upper limb & Subcutaneous & 2 & B & 2 \\
\hline $\mathrm{F}$ & 49 & Upper limb & Intramuscular & 1 & $\mathrm{~B}$ & 5 \\
\hline $\mathrm{F}$ & 55 & Upper limb & Subcutaneous & 2 & A & 3.4 \\
\hline M & 47 & Upper limb & Subcutaneous & 1 & A & 2.4 \\
\hline $\mathrm{F}$ & 35 & Dorsal region & Intramuscular & 2 & A & 1.5 \\
\hline M & 50 & Cervical region & Subcutaneous & 1 & $\mathrm{~B}$ & 2.4 \\
\hline
\end{tabular}


Tabella 1 Caratteristiche dei pazienti e delle lesioni

\begin{tabular}{|c|c|c|c|c|c|c|}
\hline Sesso & Età & Sede anatomica & Localizzazione & Pattern & Sottogruppo & Diametro $(\mathrm{cm})$ \\
\hline$F$ & 32 & Arto inferiore & Inframuscolare & 2 & $A$ & 1,5 \\
\hline$F$ & 55 & Arto inferiore & Inframuscolare & 2 & $B$ & 4 \\
\hline$M$ & 61 & Arto inferiore & Inframuscolare & 1 & $B$ & 4,5 \\
\hline$F$ & 37 & Arto inferiore & Inframuscolare & 1 & $B$ & 1,5 \\
\hline$F$ & 35 & Arto inferiore & Inframuscolare & 2 & $B$ & 6 \\
\hline$F$ & 45 & Arto inferiore & Inframuscolare & 1 & $A$ & 1 \\
\hline$F$ & 45 & Arto inferiore & Inframuscolare & 1 & $A$ & 3 \\
\hline$M$ & 90 & Arto inferiore & Sottocutaneo & 2 & $B$ & 2,5 \\
\hline$F$ & 58 & Arto inferiore & Inframuscolare & 1 & $B$ & 3 \\
\hline$F$ & 58 & Arto inferiore & Inframuscolare & 1 & $B$ & 4 \\
\hline$M$ & 68 & Arto inferiore & Inframuscolare & 1 & $B$ & 3 \\
\hline$M$ & 50 & Arto inferiore & Inframuscolare & 1 & $A$ & 2,5 \\
\hline$M$ & 64 & Arto inferiore & Inframuscolare & 1 & $B$ & 3,5 \\
\hline$F$ & 23 & Arto inferiore & Inframuscolare & 1 & $A$ & 2,4 \\
\hline$M$ & 37 & Arto inferiore & Sottocutaneo & 1 & $A$ & 2,3 \\
\hline$F$ & 55 & Arto inferiore & Inframuscolare & 2 & $A$ & 2,5 \\
\hline$M$ & 72 & Arto inferiore & Inframuscolare & 2 & $B$ & 4,3 \\
\hline$F$ & 30 & Arto inferiore & Sottocutaneo & 1 & $B$ & 4 \\
\hline$F$ & 66 & Arto inferiore & Sottocutaneo & 1 & $A$ & 3 \\
\hline$M$ & 40 & Arto inferiore & Inframuscolare & 1 & $B$ & 1,2 \\
\hline$M$ & 22 & Arto inferiore & Sottocutaneo & 2 & $B$ & 3,4 \\
\hline$M$ & 56 & Arto inferiore & Inframuscolare & 1 & $B$ & 2,3 \\
\hline$F$ & 27 & Arto inferiore & Sottocutaneo & 2 & $A$ & 2,4 \\
\hline$M$ & 80 & Arto inferiore & Sottocutaneo & 1 & $B$ & 1,1 \\
\hline$F$ & 19 & Arto inferiore & Sottocutaneo & 2 & $B$ & 3 \\
\hline$F$ & 22 & Arto inferiore & Inframuscolare & 2 & $B$ & 4,1 \\
\hline$M$ & 43 & Arto inferiore & Inframuscolare & 2 & $B$ & 2 \\
\hline$M$ & 46 & Arto inferiore & Sottocutaneo & 1 & $B$ & 3,5 \\
\hline$M$ & 69 & Arto inferiore & Sottocutaneo & 1 & $B$ & 2,5 \\
\hline$F$ & 29 & Arto inferiore & Inframuscolare & 1 & $A$ & 2 \\
\hline$F$ & 52 & Arto inferiore & Inframuscolare & 1 & $A$ & 7 \\
\hline$F$ & 64 & Arto inferiore & Inframuscolare & 1 & $B$ & 0,9 \\
\hline$M$ & 40 & Arto inferiore & Sottocutaneo & 1 & $B$ & 3 \\
\hline$F$ & 37 & Arto inferiore & Sottocutaneo & 1 & $A$ & 3,6 \\
\hline$M$ & 67 & Arto inferiore & Sottocutaneo & 1 & $B$ & 4 \\
\hline$F$ & 39 & Arto superiore & Inframuscolare & 1 & $B$ & 4 \\
\hline$M$ & 48 & Arto superiore & Inframuscolare & 1 & $B$ & 7 \\
\hline$F$ & 15 & Arto superiore & Inframuscolare & 2 & $A$ & 2 \\
\hline$F$ & 26 & Arto superiore & Inframuscolare & 1 & $A$ & 1 \\
\hline$M$ & 34 & Arto superiore & Inframuscolare & 2 & $A$ & 2,5 \\
\hline$M$ & 32 & Arto superiore & Inframuscolare & 1 & $A$ & 2 \\
\hline$F$ & 43 & Arto superiore & Inframuscolare & 1 & $A$ & 4 \\
\hline$M$ & 48 & Arto superiore & Inframuscolare & 1 & $A$ & 2 \\
\hline$F$ & 73 & Arto superiore & Inframuscolare & 1 & $A$ & 5 \\
\hline$F$ & 51 & Arto superiore & Sottocutaneo & 1 & $B$ & 1 \\
\hline$M$ & 54 & Arto superiore & Inframuscolare & 1 & $A$ & 7 \\
\hline$F$ & 26 & Arto superiore & Inframuscolare & 1 & $B$ & 3 \\
\hline$F$ & 58 & Arto superiore & Sottocutaneo & 1 & $A$ & 2,5 \\
\hline$F$ & 46 & Arto superiore & Sottocutaneo & 2 & $B$ & 2,1 \\
\hline$F$ & 48 & Arto superiore & Sottocutaneo & 1 & $B$ & 1,5 \\
\hline$M$ & 65 & Arto superiore & Inframuscolare & 2 & $B$ & 4 \\
\hline$F$ & 47 & Arto superiore & Inframuscolare & 1 & $B$ & 2,9 \\
\hline$M$ & 63 & Arto superiore & Sottocutaneo & 1 & $A$ & 2,7 \\
\hline$F$ & 43 & Arto superiore & Sottocutaneo & 2 & $B$ & 1,4 \\
\hline$M$ & 51 & Arto superiore & Inframuscolare & 2 & $B$ & 0,9 \\
\hline$F$ & 32 & Arto superiore & Sottocutaneo & 2 & $B$ & 2 \\
\hline$F$ & 49 & Arto superiore & Inframuscolare & 1 & $B$ & 5 \\
\hline$F$ & 55 & Arto superiore & Sottocutaneo & 2 & $A$ & 3,4 \\
\hline$M$ & 47 & Arto superiore & Sottocutaneo & 1 & $A$ & 2,4 \\
\hline$F$ & 35 & Dorso & Inframuscolare & 2 & $A$ & 1,5 \\
\hline$M$ & 50 & Regione cervicale & Sottocutaneo & 1 & $B$ & 2,4 \\
\hline
\end{tabular}


a $5 \mathrm{cc}$ saline flush. The patients were not required either to fast or to undergo any particular laboratory tests. In no cases were any adverse events observed. At the end of the injection, a timer was started that allowed determination of the time elapsed between the entry of the contrast medium in the circulation and its arrival in the lesion. The time span considered for the arterial phase ranged from 0 to $25 \mathrm{~s}$ from injection completion. Beyond this point, it was considered as having entered the venous phase. The presence of hyperechoic intraparenchymal spots testified to contrast medium arrival in the lesion. Contrast medium time of arrival and distribution pattern was documented on video clips, each lasting about $30 \mathrm{~s}$ and which highlighted the most significant moments of the study. All lesions were studied for at least $3 \mathrm{~min}$ (late phase).

\section{Results}

The examinations were carried out by a pool of colleagues experienced in US imaging and following the technique outlined above. Images were then reviewed by two radiologists who performed the examinations in $88.5 \%(54 / 61)$ of cases. There was no disagreement in image interpretation. The neurinomas examined at baseline US exhibited a predominantly fusiform appearance (40/61), with the nerves from which they had arisen being visible at either pole (37/61). Sometimes, they appeared as rounded (14/61) or as lobular (7/61), and it was not possible to visualise the nerve from which they had originated (23/61). Often, anechoic areas were observed inside them (22/61), and, more rarely, (3/61) intralesional calcification was seen.

After US contrast agent administration, neurinomas showed the following characteristics: in $41 / 61$ cases, the contrast material distributed from the periphery to the centre, with an interweaving of blood vessels of varying thickness - some straight, others tortuous - leaving spaces of varying size without visible blood vessels. This distribution pattern was termed reticular (pattern 1). Depending on blood vessel diameter and size of spaces interposed between them, two further subtypes were also recognised: one (18/41) with a looser mesh, which we termed looseknit reticular (pattern 1A), and the second with a denser mesh (in 23/41 cases), which we named tight-knit reticular (pattern 1B) (Fig. 1). In 20/61 lesions, however, the contrast agent did not follow a reticular distribution but diffused within the parenchyma, with densely packed, homogeneously distributed blood vessels, except for the presence of one or more circumscribed areas that exhibited no contrast enhancement. This subtype was termed diffuse heterogeneous enhancement (pattern type 2) and was further divided into two subtypes based, in $7 / 21$ cases, on the presence of a single unenhancing area (pattern $2 \mathrm{~A}$ ), or with more than con 5 cc di soluzione fisiologica; ai pazienti non sono stati richiesti digiuno o particolari esami di laboratorio; in tutti i casi non si sono mai verificati eventi avversi. A fine iniezione viene fatto partire un timer che consente di valutare il tempo che intercorre tra il passaggio in circolo del contrasto e l'arrivo dello stesso nella lesione esaminata. Il range temporale considerato per la fase arteriosa va da 0 a 25 secondi dalla fine dell'iniezione; oltre è considerato fase venosa. La presenza di spot iperecogeni intraparenchimali dimostra l'arrivo del MdC nella lesione; il tempo di arrivo e la modalità di distribuzione sono documentate mediante la registrazione di videoclips, ciascuno della durata di circa 30 secondi, che evidenziano i momenti più significativi dello studio. Tutte le formazioni sono state studiate per almeno tre minuti (fase tardiva).

\section{Risultati}

Gli esami sono stati eseguiti da un pool di colleghi esperti della metodica, secondo le indicazioni precedentemente menzionate, ed in seguito revisionati esclusivamente da due di essi, che sono risultati materialmente gli esecutori dell'esame nel $88,5 \%$ dei casi (54/61 casi). L'analisi è sempre risultata consensuale. I neurinomi esaminati, nello studio in basale, hanno presentato prevalentemente un aspetto fusiforme (40/61) ai cui poli si riconosceva la struttura nervosa dalla quale si sviluppavano (37/61); talvolta apparivano rotondeggianti (14/61) o bozzuti (7/61) e non si è riusciti a visualizzare la struttura nervosa di appartenenza (23/61). Spesso, nel loro contesto si sono osservate aree anecogene (22/61); più raramente, in 3/61 casi, presentavano calcificazioni intralesionali.

Dopo somministrazione di MdC ecografico, i 61 neurinomi esaminati hanno presentato le seguenti caratteristiche: in 41/61 casi il MdC si distribuiva dalla periferia al centro, con un intreccio di vasi, costituito da linee più o meno spesse, a decorso tortuoso o rettilineo, che si intersecano lasciando spazi di differenti dimensioni privi di vasi. Tale distribuzione da noi è stata definita "a reticolo" (pattern 1). In base all'entità dei vasi ed alla grandezza degli spazi fra questi interposti sono stati, inoltre, riconosciuti due sottotipi: uno con aspetto di incrocio più rado in 18/41 casi, definito "reticolo a maglie larghe" (pattern 1A) e uno con aspetto di incrocio dei vasi più fitto in 23/41 casi, definito "reticolo a maglie strette" (pattern 1B) (Fig. 1). In 20/61 lesioni, invece, il MdC non seguiva una distribuzione di tipo a reticolo, ma si diffondeva all'interno del parenchima con vasi fitti, omogeneamente distribuiti, eccezion fatta per la presenza di una o più aree circoscritte prive di enhancement. Tale quadro è stato da noi denominato "impregnazione diffusa disomogenea" (pattern 2) e suddiviso in due sottotipi in base alla presenza di un'area priva di enhancement (pattern 2A) in 7/21 casi o più di un'area avascolare (pattern 2B) in 13/21casi (Fig. 2). In tutti i 61 casi l'osservazione è stata protratta fino a tre minuti dall'iniezione per studiare 

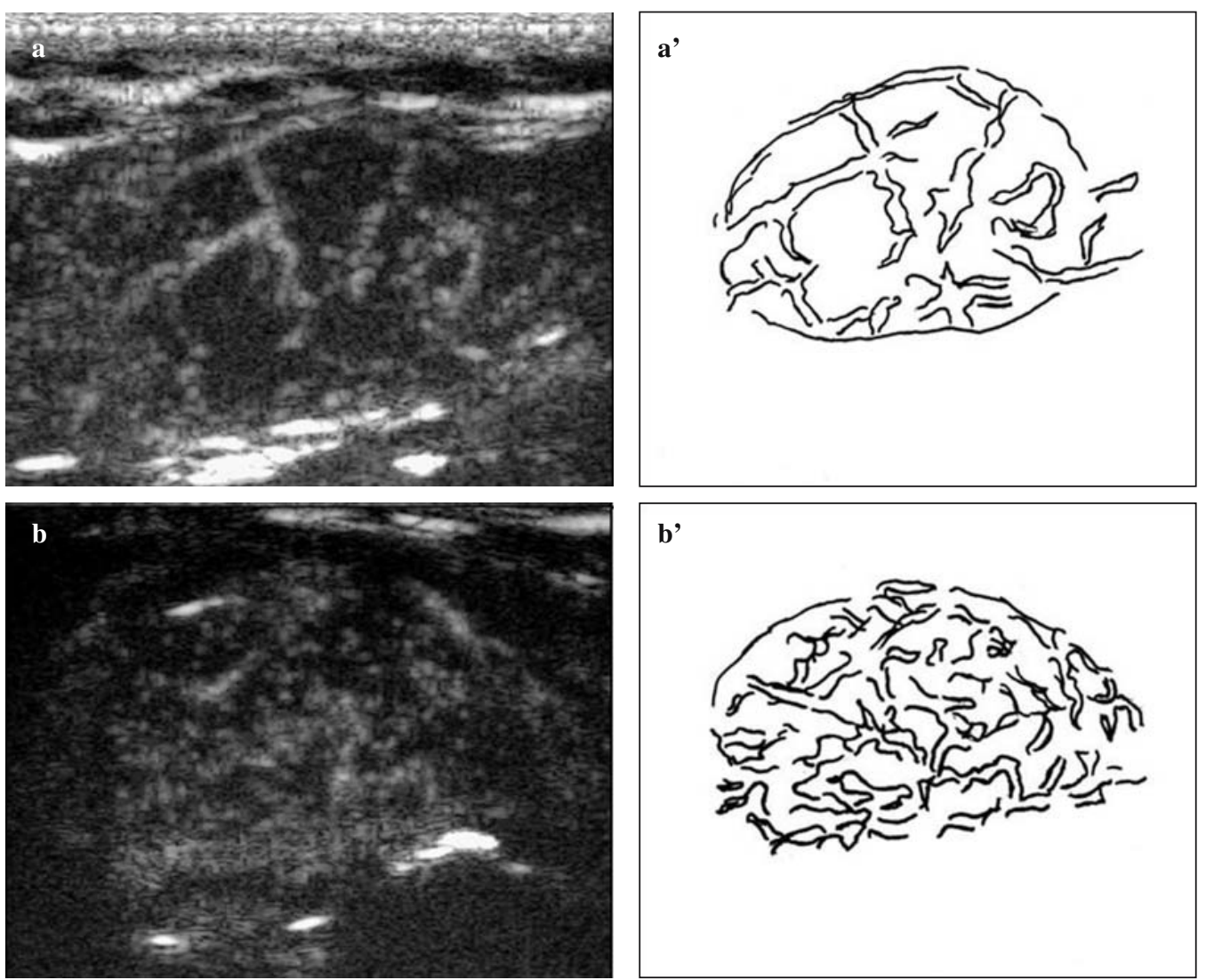

Fig.1a,b Longitudinal sections of two lesions studied with an 8-3 MHz LA532 linear probe after intravenous administration of ultrasound contrast material: example of patterns 1A (a) loosely-knit reticular and 1B (b) tightly-knit reticular. The drawings alongside a and b clarify vessel distribution, with the characteristic vascular tangle termed by us as reticular, the appearance of which, in presenting a variable tightness of their mesh, can be used to distinguish the two subgroups.

Fig. 1a,b Due differenti lesioni studiate mediante scansioni longitudinali dopo somministrazione endovena di MdC ecografico, con sonda lineare LA532 8-3 MHz: esempio di pattern 1A (a) "reticolo a maglie larghe" e di pattern 1B (b) "reticolo a maglie strette". I disegni a fianco (a' e b'), chiariscono la distribuzione dei vasi, con il caratteristico intreccio vascolare definito da noi "a reticolo", il cui aspetto, se più rado o più fitto, permette di contraddistinguere i due sottogruppi.

one avascular area (pattern 2B), in 13/21 cases (Fig. 2). In all 61 cases, the observation continued until at least $3 \mathrm{~min}$ after contrast injection in order to study lesion enhancement pattern during the late venous phases as well.

Analysis demonstrated that $67.2 \%$ of neurinomas exhibited the reticular enhancement (pattern 1), whereas the remaining 32.8\% showed diffuse heterogeneous enhancement (pattern 2) (Table 1). We noted that the lesions showing reticular enhancement (pattern 1, subtype A or B) tended to exhibit diffuse and homogeneous distribution of contrast medium during the late venous phases, whereas all neurinomas with diffuse heterogeneous enhancement (pattern 2) maintained the same appearance even $3 \mathrm{~min}$ after the injection.

\section{Discussion}

According to the international literature, neurinomas, or il comportamento delle lesioni anche nei tempi venosi tardivi.

Dall'analisi percentuale risulta pertanto che il $67,2 \%$ dei neurinomi presenta un pattern di tipo 1, "a reticolo", mentre il 32,8\% presenta un pattern di tipo 2 "a impregnazione diffusa disomogenea" (Tabella 1). Abbiamo infine notato che le lesioni che presentavano un pattern 1 "a reticolo", sia di tipo A che di tipo B, nelle fasi venose tardive tendevano ad una diffusa ed omogenea distribuzione del contrasto all'interno della lesione, mentre tutti i neurinomi che appartenevano al gruppo con pattern di tipo 2 "impregnazione diffusa disomogenea" mantenevano lo stesso aspetto anche a tre minuti dall'iniezione.

\section{Discussione}

I neurinomi, o schwannomi nella letteratura internazionale, sono tumori benigni a partenza dalla guaina nervosa; sono riconoscibili come una massa fusiforme disposta eccentrica- 

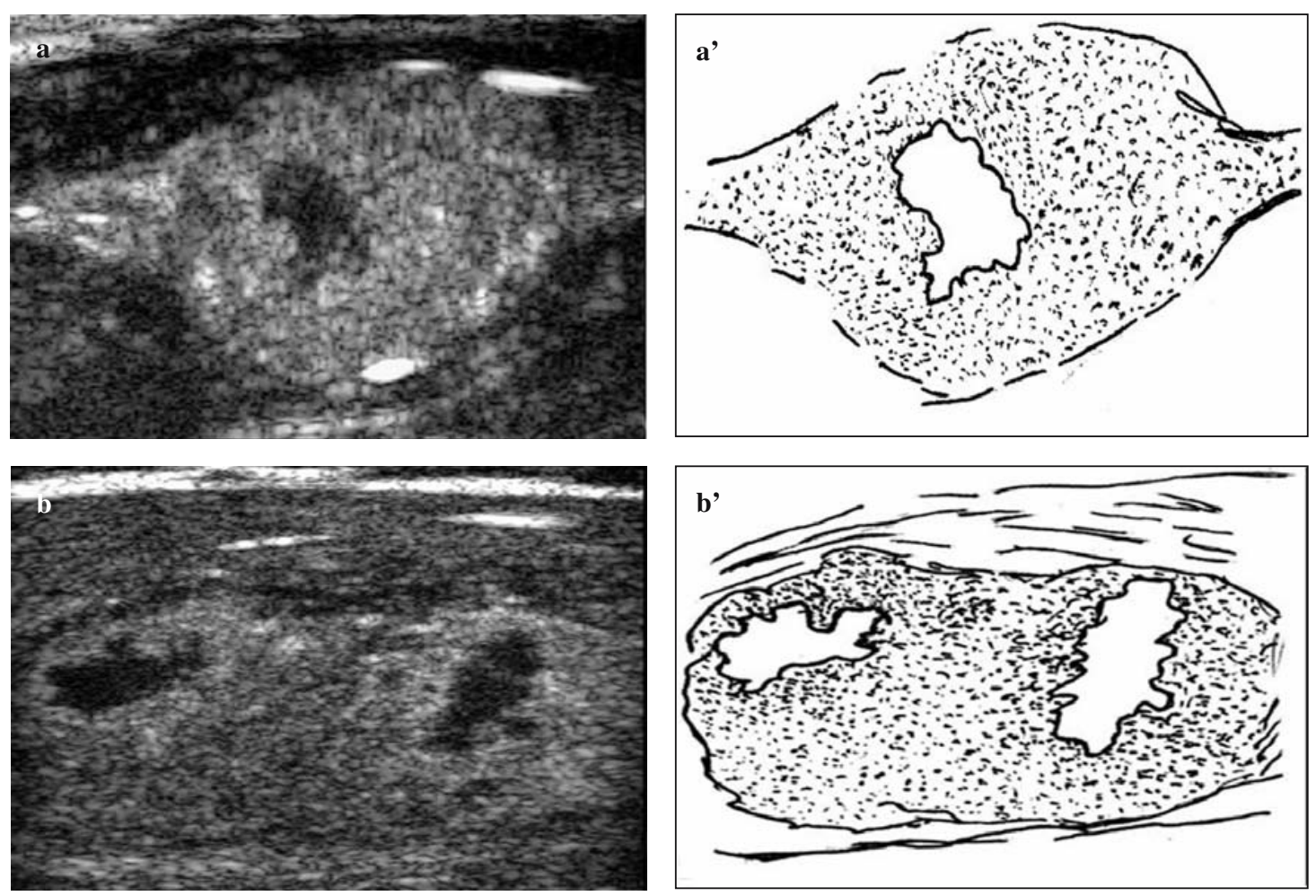

Fig. 2a,b Longitudinal sections of two lesions studied with an 8-3 MHz LA532 linear probe after intravenous administration of ultrasound contrast agent: example of diffuse heterogeneous enhancement, pattern 2A (a) showing the presence of a single nonenhancing area and pattern 2B (b) due to the presence of several nonenhancing areas. The drawings alongside $\mathbf{a}$ and $\mathbf{b}$ clarify contrast agent diffusion within the lesions' densely and evenly distributed blood vessels, with the exception of the avascular areas.

Fig. 2a,b Due differenti lesioni studiate mediante scansioni longitudinali dopo somministrazione endovena di MdC ecografico, con sonda lineare LA532 8-3 MHz: esempio di pattern 2A (a) "impregnazione diffusa disomogenea" per la presenza di una singola area priva di enhancement e di pattern $2 B$ (b) "impregnazione diffusa disomogenea" per la presenza di più aree prive di enhancement. A fianco, i disegni (a' e b') chiariscono la diffusione del MdC all'interno della lesione attraverso vasi fitti e omogeneamente distribuiti ad eccezione delle aree avascolari.

schwannomas, are benign tumours originating in the nerve sheath and are recognisable as fusiform masses arranged eccentrically to the nerve and surrounded by a capsule known as the epineurium. The presence of the capsule allows for complete surgical excision of the lesion. A location eccentric to the nerve fibres is indicative for a diagnosis of neurinoma, but this finding is not very frequent, and the presence of intralesional cystic spaces is typical of cystic neurinomas but very rare in neurofibromas. These characteristics are not constant, but their identification allows for correct diagnosis of a neurinoma. In doubtful cases, due to the absence of a characteristic US morphology or typical symptoms or signs, such as the electric shock, the diagnosis is less straightforward.

With the use of modern technology, valuable information can be obtained with regard to blood vessel distribution within the lesion [17-20] by using the maximum harmonic signal generated by microbubble contrast agents when sonicated with a frequency around 3-3.5 MHz. Our research aimed to investigate this aspect, in part in consideration of the fact that few studies have evaluated the vascular appearance of neurinomas and those that have mente al nervo e circondata da una capsula detta epinevrio. La presenza della capsula consente la completa asportazione chirurgica della lesione. La localizzazione eccentrica rispetto alle fibre nervose è suggestiva per la diagnosi di neurinoma, ma questo riscontro non è così frequente; la presenza di lacune cistiche intralesionali è tipica dei neurinomi, molto rara nei neurofibromi. Questi aspetti non sono costanti, tuttavia il loro riscontro consente una corretta diagnosi di neurinoma. Nei casi dubbi, per la mancanza della caratteristica morfologia ecografica o della tipica sintomatologia, tipo scossa elettrica, la diagnosi risulta meno agevole.

Attualmente, grazie all'impiego dei moderni ritrovati tecnologici, è possibile aggiungere preziose informazioni circa la distribuzione dei vasi all'interno della lesione [17-20], sfruttando il massimo segnale armonico proveniente dalle microbolle del mezzo di contrasto ecografico, quando insonate da un fascio ultrasonoro vicino ai 3-3,5 $\mathrm{MHz}$. La nostra ricerca si inserisce in questa prospettiva, anche in considerazione del fatto che gli studi in letteratura internazionale, riguardanti la valutazione dell'aspetto vascolare dei neurinomi, sono scarsi e riguardano esclusivamente la RM. 

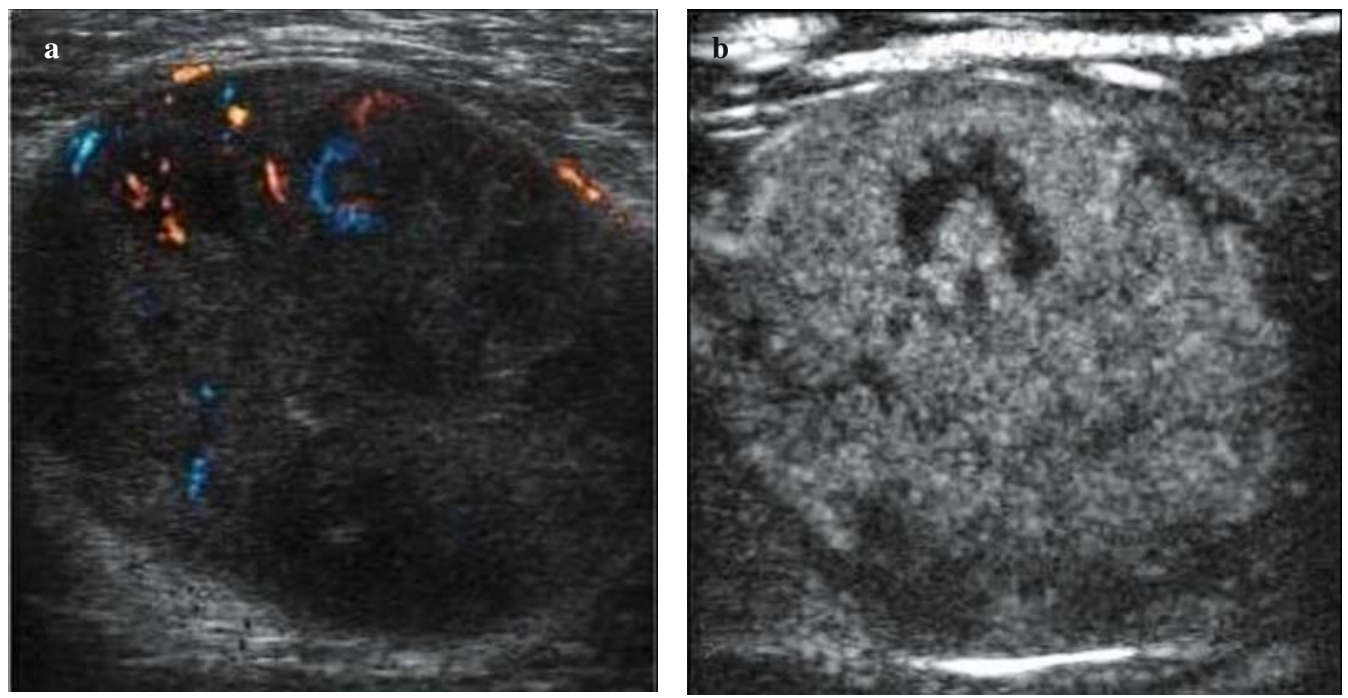

Fig. 3a,b Longitudinal sections of the same lesion studied with Power Doppler (PD) and following intravenous administration of ultrasound contrast agent, with an LA532 8-3 MHz linear probe: PD (a) depicts a few intralesional vascular spots, underestimating the lesion's true vascularity, which is demonstrated by lesion enhancement after contrast administration $(\mathbf{b})$.

Fig. 3a,b Stessa lesione studiata mediante scansioni longitudinali al PD e dopo somministrazione endovena di MdC ecografico, con sonda lineare LA532 8-3 MHz: il PD (a) visualizza pochi spot vascolari intralesionali, sottostimando la reale vascolarizzazione della lesione, dimostrata dall'enhancement della lesione dopo contrasto (b).

were conducted with magnetic resonance imaging.

US imaging performed with machines equipped with high-frequency linear-array probes designed to be used with contrast-specific software allows accurate assessment of the passage of the contrast microbubbles within even the smallest blood vessels, so the findings are far more specific and precise than evaluation of macrovasculature with power Doppler imaging [21-23] (Fig. 3). This enabled the identification of characteristic patterns within the group of neurinomas assessed in our study. The added value of US contrast agents is related to the precise evaluation of the distribution of both large and small blood vessels, which allows differentiation into the two main subtypes: reticular, and diffuse heterogeneous enhancement. The recognition of either pattern, associated with the morphological characteristics of the lesion, proved useful in directing us towards the diagnosis.

\section{Conclusions}

The results obtained in this study show that all neurinomas examined could be divided into two groups according to the type of vascular pattern observed: reticular, or diffuse heterogeneous enhancement. From our experience, it can be stated that a thorough baseline US examination, aimed at identifying the morphostructural characteristics of neurinomas and visualising the possible continuity with the nerve fibres, is suggestive for the diagnosis of a neuri-
Lo studio ecografico, effettuato con apparecchiature dotate di sonde lineari ad alta frequenza dedicate all'utilizzo di software contrasto specifico, consente una precisa valutazione del passaggio delle microbolle all'interno dei vasi, anche di piccole dimensioni; il riscontro è altamente più specifico e preciso rispetto alla valutazione della macrovascolarizzazione effettuata con il PD (Fig. 3) [21-23]. Ciò ha permesso l'identificazione di pattern caratteristici all'interno del gruppo di neurinomi preso in esame. Il valore aggiunto del mezzo di contrasto ecografico è dato dalla precisa valutazione della distribuzione dei vasi sia di grandi che di piccole dimensioni, in base alla quale sono stati differenziati due principali pattern di distribuzione: a reticolo e a impregnazione diffusa disomogenea. Il riconoscimento di uno dei due pattern, associato alla caratteristiche morfologiche della lesione, è utile ad indirizzarci verso il sospetto diagnostico.

\section{Conclusioni}

Dai risultati ottenuti in questo studio si evince che tutti $i$ neurinomi analizzati possono essere distinti in due gruppi, in base al tipo di pattern vascolare riscontrato: di tipo reticolare o ad impregnazione diffusa disomogenea. Dall'esperienza acquisita possiamo affermare che un accurato esame ecografico di base, mirato alla ricerca delle caratteristiche morfo-strutturali dei neurinomi e dell'eventuale visualizzazione della continuazione delle fibre nervose è suggestiva per la diagnosi di neurinoma; se a questo segue poi lo studio con MdC, il riscontro della tipica distribuzione della vasco- 
noma. If this is followed up by a contrast-enhanced US examination, the finding of a characteristic vascular pattern in the two subtypes identified as reticular (either loose knit or tight knit) and diffuse heterogeneous enhancement (due to the presence of one or more nonenhancing areas) assists in characterising these masses, thus increasing the operator's diagnostic confidence. This is particularly useful in doubtful cases, especially in lesions that do not exhibit the typical clinical and morphological features of neurinomas and that may mimic more aggressive lesions. larizzazione nei due pattern identificati come "reticolo" ("a maglie larghe" o "a maglie strette") e "impregnazione diffusa disomogenea" (per la presenza di "una" o "più aree prive di enhancement") contribuisce a riconoscere come tali queste formazioni, aumentando così la confidenza diagnostica dell'operatore. Tutto questo si dimostra particolarmente utile nei casi dubbi, soprattutto in quelle lesioni che non presentano caratteristiche morfologiche e cliniche tipiche dei neurinomi, e che possono simulare lesioni di natura aggressiva.

\section{Conflict of interest None}

\section{References/Bibliografia}

1. De Marchi A, De Petro P, Pozza S et al (2004) Apparato muscoloscheletrico. In: Rossi S, Calliada F, Martegani A (eds) Mezzi di contrasto in ecografia. Testo atlante. Poletto editore, Gudo Visconti, Milano, pp 256-261

2. Beaman FD, Kransdorf MJ, Menke DM (2004) Schwannoma: radiologicpathologic correlation. Radiographics 24:1477-1481

3. Simonovsky V (1997) Peripheral nerve schwannoma preoperatively diagnosed by sonography: report of three cases and discussion. Eur J Radiol 25:47-51

4. Beggs I (1997) Pictorial review: imaging of peripheral nerve tumours. Clin Radiol 52:8-17

5. Martinoli C, Bianchi S, Dahmane M et al (2002) Ultrasound of tendons and nerves. Eur Radiol 12:44-55

6. De Marchi A, Verga L, Pozza S et al (2005) I tumori dei tessuti molli. In: Faletti C, Masciocchi C (eds) Trattato di diagnostica per immagini nella patologia muscoloschelerica. UTET, Torino, pp 141-159

7. Monetti (2000) Ecografia muscolo tendinea. Imaging integrato. IdelsonGnocchi, Napoli

8. Kuo YL, Yao WJ, Chiu HY (2005) Role of sonography in the preoperative assessment of neurilemmoma. J Clin Ultrasound 33:87-89

9. Beggs I (1999) Sonographic appearances of nerve tumours. J Clin Ultrasound 27:363-368
10. Bendix N, Wolf C, Gruber H, Bodner G (2005) Pictorial essay: Ultrasound of tumours and tumour-like lesions of peripheral nerves. Ultraschall Med 26:318-324

11. Burns PN, Wilson SR (2006) Microbubble contrast for radiological imaging: principles. Ultrasound Q 22:5-13

12. Burns PN, Powers J, Hope UD, Simpson FVT (1996) Harmonic imaging: principles and preliminary results. Angiology 47:S63-S74

13. Ricci P, Laghi A, Cantisani V et al (2005) Contrast-enhanced sonography with SonoVue: enhancement patterns of benign focal liver lesions and correlation with dynamic gadobenate dimeglumine-enhanced MRI. AJR Am J Roentgenol 184:821-827

14. Piscaglia F, Lencioni R, Sagrini E et al (2010) Characterization of focal liver lesions with contrast-enhanced ultrasound. Ultrasound Med Biol 36:531-550

15. Sorelli PG, Cosgrove DO, Svensson WE et al (2010) Can contrast-enhanced sonography distinguish benign from malignant breast masses? J Clin Ultrasound 38:177-181

16. Amoretti N, Grimaud A, Hovorka E et al (2006) Peripheral neurogenic tumours: is the use of different types of imaging diagnostically useful? Clinical Imaging 30:201-205
17. Schlief R, Bauer A (1996) Ultrasound contrast media. New perspectives in ultrasound diagnosis. Radiologe 36:51-57

18. Ricci P, Cantisani V, Ballesio L et al (2007) Benign and malignant breast lesions: efficacy of real time contrastenhanced ultrasuond vs MRI. Ultrashall Med 28:57-62

19. De Marchi A, De Petro P, Faletti C et al (2003) Echo color power Doppler with contrast medium to evaluate vascularization in lesion of the soft tissue of the limbs. Chir Organi Mov 88:225-231

20. Bartolozzi C, Crocetti L, Della Pinna MC (2007) How to differentiate liver lesions in cirrhosis. JBR-BTR 90:475481

21. Kook SH, Kwag HG (2003) Value of contrast-enhanced power Doppler sonography using a microbubble echo-enhancing agent in evaluation of small breast lesions. J Clin Ultrasound 31:227-238

22. Calliada F, Campani R, Bottinelli O et al (1998) Ultrasound contrast agents: basic principles. Eur J Radiol 27(Suppl 2):S157-S160

23. Sofka CM, Lin D, Adler RS (2005) Advantages of colour B-mode imaging with contrast optimization in sonography of low-contrast musculoskeletal lesion and structures in the foot and ankle. J Ultrasound Med 24:215-218 